\title{
Dispensing of antibiotics without prescription in Greece, 2008: another link in the antibiotic resistance chain
}

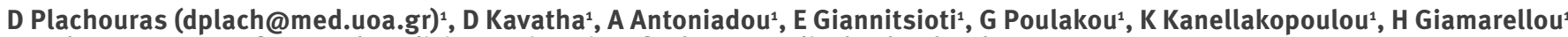
1. 4 th Department of Internal Medicine, University of Athens, Medical School, Athens, Greece

Antibiotic resistance has been associated with the use of antibiotics. The dispensing of antimicrobials without prescription is a potential source of inappropriate antibiotic use. In our study, antibiotics were requested without prescription from pharmacies in the metropolitan area of Athens in Greece in 2008. Twenty-one collaborators visited 174 pharmacies and asked for either amoxicillin/clavulanate acid or ciprofloxacin without providing a prescription or any other justification for the request. In Greece additional restrictions for fluoroquinolone prescriptions were implemented in 2003 after which a separate specific prescription form needs to be filled in by the prescriber, justifying the choice of any fluoroquinolone. Amoxicillin/clavulanate acid was dispensed in all cases. Furthermore, despite the regulation restricting the prescription of ciprofloxacin, this drug was dispensed by $53 \%$ of the pharmacies. It appears that the implementation of measures to restrict the use of certain antibiotics (e.g. ciprofloxacin that was studied in our case) was effective in reducing, although not eliminating, inappropriate dispensing. Overall, dispensing of antimicrobials without prescription is a widespread practice in the studied area and is contributing to the overuse of antibiotics.

\section{Introduction}

Antibiotic resistance is a major public health threat worldwide. There is plenty of evidence that the use of antibiotics is associated with the emergence of resistance [1]. Rates of antibiotic consumption correlate closely with rates of antimicrobial resistance at national level, including both the hospital environment and outpatient settings [2,3]. Variation in resistance correlates with variability in antibiotic use across Europe $[4,5]$. This difference reflects socio-economic, healthcare system, legislative and cultural differences among European countries [6]. To a large extent, this variation is a result of the over- and misuse of antibiotics, especially for upper respiratory tract infections, many of which are of viral cause. Excessive use is partly attributed to inappropriate prescriptions by physicians. However, self-medication with antibiotics is also a known practice in various European countries, especially in the southern and eastern parts [6,7], and this may exacerbate the problem $[6,7]$. The two main sources of self-medication include the use of left-over antibiotics from previous courses of treatment and the acquisition from pharmacies without prescription [8].

In Greece, both the use of antibiotics and resistance rates are high, including resistance to penicillin and macrolides among community-acquired isolates of Streptococcus pneumoniae [9] and Streptococcus pyogenes [10]. Since 1950, national law forbids the dispensing of antibiotics 'over the counter' without a prescription. Additionally, since 2003 and according to an official decision by the Ministry of Health, a separate specific prescription form needs to be filled in by the prescriber, justifying the choice of any fluoroquinolone or third generation cephalosporin prescribed orally, before the drug is dispensed. This measure was implemented in an effort to preserve the efficacy of these classes of antimicrobials. However, there is no control of the actual practice and in effect the law is not implemented. It is generally accepted by the public that antibiotics can be acquired from pharmacies without prescription, either by direct purchase or by retrospectively providing a prescription. The present study was undertaken in order to quantify the extent of this practice.

\section{Methods}

Pharmacies in the greater Athens metropolitan area, were visited from April to May 2008 by 21 voluntary collaborators, who asked for either ciprofloxacin or amoxicillin/clavulanate acid without providing a prescription or any other relevant justification. Amoxicillin/clavulanate acid was chosen for our study because it is one of the most frequently used antibiotics and ciprofloxacin because of the additional restrictions in dispensing for fluoroquinolones in Greece. The collaborators were advised to neither insist in case the antibiotic was refused, nor to simulate any specific disease or symptom, in order not to influence the pharmacist in the decision. Collaborators included physicians, nurses and laboratory technicians, but the identity or occupation was not revealed during the visit. For the 
purposes of the study, the greater Athens area was divided in four main regions of comparable size - centre, northern suburbs, western suburbs and southern suburbs with respective populations of 745,000 , 594,000, 469,000 and 784,000 people, according to a 2001 census [11]. The total number of pharmacies in the greater Athens area was 3,426 in 2006 according to the most recent available data from the national statistical service of Greece [11]. The number of pharmacies visited in each area was chosen to roughly reflect the population. Each collaborator was assigned a specific area and the pharmacies visited were chosen randomly by the collaborator. All pharmacies visited were privately owned small enterprises. During the visit there was no way of differentiating between pharmacy employees and pharmacist, and the voluntary collaborators did not attempt to do this as it might have compromised the aim of the study. The collaborators purchased the antibiotic and the number of pharmacies that dispensed the antibiotics was recorded. In addition the reason for refusing sale of the requested drugs and any comments made by the pharmacist, were recorded. The difference between dispensing the non-restricted (amoxicillin/clavulanate acid) and the restricted (ciprofloxacin) antibiotic was compared using the Fisher's exact test.

\section{Results}

One hundred and seventy-four visits in different pharmacies were performed, covering $5 \%$ of the 3,426 pharmacies in the greater Athens area. Ciprofloxacin was requested during 102 visits and it was dispensed by 54 pharmacies (53\%). Amoxicillin/clavulanate acid was requested during 72 visits and it was dispensed in all cases $(100 \%)$. The difference between restricted and non-restricted antibiotics was significant ( $p<0.001)$. Antibiotic dispensing in different areas of Athens is shown in the Table. Dispensing practices did not differ significantly in the various areas.

In 107 (85\%) of the 126 visits where the antibiotic was sold without prescription, no comment was made by the pharmacist and no reason for the intended antibiotic use was requested. In all 48 cases where dispensing of ciprofloxacin was refused, the reason offered by the pharmacist was the requirement of the special prescription form for fluoroquinolones. In one case the

\section{TABLE}

Dispensing of ciprofloxacin without prescription in pharmacies by areas in Athens, Greece, April-May 2008 $(\mathrm{n}=54)$

\begin{tabular}{|l|c|c|}
\hline & $\begin{array}{c}\text { Number of } \\
\text { pharmacies visited }\end{array}$ & $\begin{array}{c}\text { Number of pharmacies } \\
\text { dispensing ciprofloxacin } \\
(\%)\end{array}$ \\
\hline Western suburbs & 26 & $14(54)$ \\
\hline Northern suburbs & 22 & $11(50)$ \\
\hline City centre & 26 & $12(46)$ \\
\hline Southern suburbs & 28 & $17(61)$ \\
\hline Total & 102 & $54(53)$ \\
\hline
\end{tabular}

pharmacist refused ciprofloxacin and offered amoxicillin/clavulanate acid as an alternative. In three (6\%) of the cases where ciprofloxacin was sold, the pharmacist informed the collaborator that "normally a physician's prescription is required". The same occurred only once when amoxicillin/clavulanate acid was requested. During another visit the pharmacy employee initially refused to dispense ciprofloxacin but the drug was consequently dispensed by the pharmacist. In only three cases of amoxicillin/clavulanate acid dispensing the collaborator was informed by the pharmacist about adverse events - mainly allergies and diarrhoea - or asked whether such events had occurred in the past when the buyer had used antibiotics. In three other cases information on dosage was provided by the pharmacist.

\section{Discussion}

The results of this study indicate that antibiotics can be very easily bought in Greek pharmacies without prescription. No pharmacist refused to dispense amoxicillin/clavulanate acid without prescription, and none asked for any justification for the purchase. Even ciprofloxacin, that is supposed to be restricted and for which a special prescription form needs to be filled in, in order for it to be dispensed, was freely dispensed in more than half of the pharmacies visited.

It appears that the implementation of measures to restrict the use of certain antibiotics (e.g. ciprofloxacin that was studied in our case) was effective in reducing, although not eliminating, inappropriate dispensing. It is notable that the only reason offered by the pharmacists for not selling ciprofloxacin, was the requirement of the special fluoroquinolone prescription form, implemented in Greece. The latter finding may well justify extending this measure to all classes of antibiotics, in addition to implementing measures aimed at curbing antibiotic dispensing without prescription.

The present results complement those of another Greek study, that included visits in pharmacies in north-western Greece by actors simulating rhinosinusitis and asking for antibiotics [12]. In that study 69 to $86 \%$ percent of the pharmacists offered an antibiotic. In our study no justification for the request of antibiotics was given by the researchers and specific antibiotics were requested.

In contrast to previous studies, where actors simulating patients made visits to the pharmacies [12-14], antibiotics were asked for and purchased without any justification whereas in the present study the collaborators avoided simulating specific symptoms or diseases. Although this is not the only way that antibiotics are requested at the pharmacy, it indicates the ease of obtaining antibiotics without prescription, as if antibiotics were everyday commodities.

The amount of antibiotics dispensed without prescription in Greece is not known. From a large public 
survey with personal interviews conducted in 2007 that included over 1,000 individuals in Greece (Giamarellou, personal communication), $25 \%$ of the sample used antibiotics in the last three months, the greater majority for upper respiratory tract infections. Twelve per cent of the sample admitted purchasing antibiotics without prescription. A questionnaire survey of 173 adults and 150 parents carried out in the urban area of Patras, Greece in 2003 , revealed that $74.6 \%$ of the adults consumed non-prescribed antibiotics and $22.7 \%$ of parents administered non-prescribed antibiotics to their children [15]. Furthermore, in the same study $55 \%$ of the participants admitted to using left-over antibiotics. Availability of antibiotics 'over the counter' is considered by the authors as one of the main reasons accounting for these observations. Self-medication by 'over the counter' antibiotics appears to be an extensive problem in Greece and this practice may contribute significantly to excess antibiotic use and increased antibiotic resistance.

Similar problems have been described in other European countries. In Spain 31\% of antibiotics consumed were found to be dispensed by community pharmacies without prescription, although it is not legal [16]. Even after an extensive institutional campaign launched by the Ministry of Health in 2007 aiming at reducing this practice, antibiotics were dispensed without a prescription by $79.7 \%$ of the pharmacies studied in Catalonia just one year after the campaign [13]. In Malta $19 \%$ of the participants in a study admitted to self-medication with antibiotics, which were provided without prescription by local pharmacies in $85 \%$ of cases [17]. In the United Kingdom a survey on antibiotic use included in the 2003 Office for National Statistics Omnibus Household Survey revealed that $4.8 \%$ of adults surveyed had at least once taken an antibiotic without advice from a doctor, dentist or nurse [18]. This phenomenon is not restricted to Europe. One of the largest questionnaire studies on antibiotic use carried out in 2001 in nine countries (United Kingdom, France, Belgium, Italy, Spain, Turkey, Thailand, Morocco and Colombia) indicated that antibiotics could be obtained without prescription in all countries [19]. In Brazil $58-74 \%$ of the pharmacies studied dispensed antibiotics 'over the counter' [14]. Non-prescribed antimicrobial drug use has also been described in latino communities in the Unites States [20].

Antibiotics are generally considered safe and 'over the counter' use has been advocated, for certain specific indications [21]. However, in contrast to all other groups of medicines, in addition to possible adverse effects to the consumer, antibiotics have an effect on the community, namely the emergence of resistance. In this study, information about possible adverse events to the individual was only provided by the pharmacy in a minority of cases. Important questions relevant to antibiotic resistance, including previous use of antibiotics, were not asked by the pharmacists. In addition no comment was made in most of the cases of the indication of the antibiotic dispensed, risking administration of an ineffective antibiotic and inadequate treatment. These observations indicate that the dispensing of antimicrobials at the pharmacy without prescription, at least as practiced in Greece and witnessed by the present study, may be unsafe.

The dispensing of antibiotics without prescription is not the only way of antibiotic overuse and misuse. Leftovers from previous treatment courses due to noncompletion of the previous treatment or the dispensing of excess medication, acquisition of antibiotics over the internet and inappropriate prescription by physicians for viral upper respiratory infections are further contributing factors [8]. These causes need to be addressed in order to ensure that this valuable class of medications is safeguarded.

Possible ways of intervention would include educating the public and pharmacists as well as stricter implementation of regulations already in force, especially regarding antibiotics. According to Greek legislation antibiotics are prescription-only medicines, however this law is not applied as there is neither control nor penalty for those not abiding by it. The European Union (EU) supports and encourages policies on the prudent use of antimicrobials in order to control antimicrobial resistance and recommends control measures to support the prudent use of antimicrobials, e.g. by "restricting systemic antimicrobial agents to prescription-only use" [22]. It is the responsibility of EU Member States to ensure the implementation of these strategies on a national level in order to avoid or limit microbial resistance. Eliminating the dispensing of antibiotics without prescription could be a valuable measure towards this goal.

\section{Acknowledgements}

We thank the collaborators in this study for their help in pharmacy visits (in alphabetical order):

N. Antonakos, S. Athanasia, F. Baziaka, K. Kalaitzaki, I. Karaiskos, D. Katsala, I. Katsarolis, E. Koratzanis, G. Koukos, P. Koutoukas, C. Massouras, A. Oikonomou, A. Papadopoulos, P. Panagopoulos, C. Protopappas, M. Souli, L. Sympardi, G. Tzempetzi, P. Valera

References

1. Albrich WC, Monnet DL, Harbarth S. Antibiotic selection pressure and resistance in Streptococcus pneumoniae and Streptococcus pyogenes. Emerg Infect Dis. 2004;10(3):514-7.

2. Goossens H, Ferech M, Vander Stichele R, Elseviers $M$. Outpatient antibiotic use in Europe and association with resistance: a cross-national database study. Lancet. 2005;365(9459):579-87.

3. Austin DJ, Kristinsson KG, Anderson RM. The relationship between the volume of antimicrobial consumption in human communities and the frequency of resistance. Proc Natl Acad Sci U S A. 1999;96(3):1152-6.

4. Cars O, Molstad S, Melander A. Variation in antibiotic use in the European Union. Lancet. 2001; 357(9271):1851-3. 
5. Molstad S, Lundborg CS, Karlsson AK, Cars O. Antibiotic prescription rates vary markedly between 13 European countries. Scand J Infect Dis. 2002;34(5):366-71.

6. Grigoryan L, Burgerhof JG, Degener JE, Deschepper R, Lundborg CS, Monnet DL, et al. Determinants of selfmedication with antibiotics in Europe: the impact of beliefs, country wealth and the healthcare system. J Antimicrob Chemother. 2008;61(5):1172-9.

7. Grigoryan L, Burgerhof JG, Haaijer-Ruskamp FM, Degener JE, Deschepper R, Monnet DL, et al. Is self-medication with antibiotics in Europe driven by prescribed use? J Antimicrob Chemother. 2007;59(1):152-6.

8. Grigoryan L, Haaijer-Rysjamp FM, Burgerhof JG, Mechtler R, Deschepper R, Tambic-Andrasevic A, et al. Self-medication with antimicrobial drugs in Europe. Emerg Infect Dis. 2006;12(3):452-9.

9. Poulakou G, Katsarolis I, Matthaiopoulou I, Tsiodras S, Kanavaki S, Hatzaki D, et al. Nationwide surveillance of Streptococcus pneumoniae in Greece: patterns of resistance and serotype epidemiology. Int J Antimicrob Agents. 2007;30(1):87-92

10. Souli M, Volonakis K, Kapaskelis A, Galani I, Grammelis V, Vorou R, et al. Characterisation of macrolide-non-susceptible Streptococcus pneumoniae colonising children attending day-care centres in Athens, Greece during 2000 and 2003. Clin Microbiol Infect. 2007;13(1):70-7.

11. National Statistical Service of Greece. [Internet]. Athens: 2010. Available from: URL: http://www.statistics.gr/portal/page/ portal/ESYE/PAGE-themes?p_param=A1604

12. Contopoulos-Ioannidis DG, Koliofoti ID, Koutroumpa IC, Giannakakis IA, Ioannidis JP. Pathways for inappropriate dispensing of antibiotics for rhinosinusitis: a randomized trial. Clin Infect Dis. 2001;33(1):76-82.

13. Llor C, Cots JM. The sale of antibiotics without prescription in pharmacies in Catalonia, Spain. Clin Infect Dis. 2009;48(10):1345-9.

14. Volpato DE, de Souza BV, Dalla Rosa LG, Melo LH, Daudt CA, Deboni L. Use of antibiotics without medical prescription. Braz J Infect Dis. 2005;9(4):288-91.

15. Mitsi G, Jelastopulu E, Basiaris H, Skoutelis A, Gogos C. Patterns of antibiotic use among adults and parents in the community: a questionnaire-based survey in a Greek urban population. Int J Antimicrob Agents. 2005;25(5):439-43.

16. Vaananen MH, Pietila K, Airaksinen M. Self-medication with antibiotics--does it really happen in Europe? Health Policy. 2006;77(2):166-71.

17. Borg MA, Scicluna EA. Over-the-counter acquisition of antibiotics in the Maltese general population. Int I Antimicrob Agents. 2002;20(4):253-7.

18. McNulty CA, Boyle P, Nichols T, Clappison P, Davey P. Don't wear me out--the public's knowledge of and attitudes to antibiotic use. J Antimicrob Chemother. 2007;59(4):727-38.

19. Pechere JC. Patients' interviews and misuse of antibiotics. Clin Infect Dis. 2001;33 Suppl 3:S170-3.:

20. Mainous AG zrd, Cheng AY, Garr RC, Tilley BC, Everett CJ, McKee MD. Nonprescribed antimicrobial drugs in Latino community, South Carolina. Emerg Infect Dis. 2005;11(6):883-8.

21. Reeves DS, Finch RG, Bax RP, Davey PG, Po AL, Lingam G, et al. Self-medication of antibacterials without prescription (also called 'over-the-counter' use). A report of a Working Party of the British Society for Antimicrobial Chemotherapy. J Antimicrob Chemother. 1999;44(2):163-77.

22. The Council of the European Union. Recommendation on the Prudent Use of Antimicrobial Agents in Human Medicine (2002/77/EC). Available from: http://eur-lex.europa.eu/pri/en/ oj/dat/2002/l_034/l_03420020205en00130016.pdf 